\title{
A SURVEY ON INCREMENTAL RELAYING PROTOCOLS IN COOPERATIVE COMMUNICATION
}

\author{
Rinu Titus ${ }^{1}$, Unnikrishnan $\mathbf{M}^{2}$, Premkumar C.V ${ }^{3}$ \\ ${ }^{1}$ Student, Department of Electronics, SOE CUSAT, Kerala, India \\ ${ }^{2}$ Student, Department of Electronics, SOE CUSAT, Kerala, India \\ ${ }^{3}$ Assistant Professor, Department of Electronics, SOE CUSAT, Kerala, India
}

\begin{abstract}
Cooperative communication is one of the latest techniques in wireless communication to form virtual antenna arrays. Cooperative communication is an alternative method for MIMO (Multiple input multiple output) system. This scheme assumes TDMA (Time division multiple access) channel accessing and divides the communication into two phases which includes direct transmission and retransmission by the relay terminal. A few cooperative diversity protocols, for example fixed relaying, selection relaying and incremental relaying are used in order to perform different sorts of processing by the relay terminals, and combining at the destination terminals. Each diversity protocol exhibits its own unique performance. Fixed relaying can be further classified into amplify and forward $(A F)$ relaying and decode and forward $(D F)$ relaying. In $A F$, relay users amplifies the signal and retransmits it, and in the case of $D F$, the relay users decodes and retransmits the signal. The Selection relaying is performed by taking the fading coefficient into account.

In this report, the recent works on incremental relaying protocol has been summarized. Incremental relaying is based on the feedback mechanism. In this scheme, relay users perform retransmission only if needed, based on the received SNR (Signal to noise ratio) or decoding error that occurs during direct transmission. It is used in cooperative communication in order to attain efficient use of degrees of freedom and to overcome bandwidth inefficiency. The authors have presented a number of protocols such as Incremental selection amplify and forward (ISAF), Joint incremental selection relaying (JISR), Fractional Incremental relaying (FIR), Selective Fractional Incremental relaying (SFIR) and Efficient Incremental relaying (EIR).
\end{abstract}

Keywords: Cooperative diversity, Relaying, Signal to noise ratio, Hybrid automatic repeat request, Spectral efficiency.

\section{INTRODUCTION}

In recent years the communication technology has developed exponentially in order to meet user needs. Cooperative communication is one of the latest technologies used in Wireless communication. Cooperative communication helps to achieve a very high data rate. Comprehensive theory, implementation and performances of cooperative communication and related topics is given by $\mathbf{J}$ N Laneman [1],B Zhao [2],Q.F Zhou [3], H. Long et al.[5] M M. Fareed [17], and a significant number of papers are available in this research area.

Cooperative communication establishes its implementation by providing cooperative diversity. Thus the network that uses cooperative communication is also termed as cooperative diversity network. In a cooperative communication system, each wireless user will transmit its own data and cooperatively transmits data of other users in the network. Fig-1 and Fig-2 represents a cooperative diversity network scenario. This particular technique can be used instead of Multiple Input Multiple Output (MIMO) [2], [3] technology. Cooperative communication works by creating a virtual network of nodes that are ready to cooperate. The nodes can be any wireless device such as mobile phones, laptops, etc. Each node provides its antenna to form virtual antenna networks. This technique overcomes the limitations of MIMO networks such as increased space utilization and issues related to incorporating several antennas into one system.

Relaying is used in cooperative diversity networks primarily to employ different types of processing by the relay terminals and combining at the destination terminals. These networks are built based on the classical relay channel model [4] and inspects the issue of creating and exploiting cooperative diversity by utilizing a collection of distributed antennas belonging to different terminals, each with its data to transmit. Repetition coding [5] is used in this system because of its ease of access and very low implementation complexity. Relaying protocols used in cooperativediversity networks are Fixed relaying, Selection relaying and Incremental relaying [1], [6]. This paper discusses the incremental relaying protocols in detail. Cooperative diversity scenario is divided into two phases such as Phase 1 and Phase 11. Phase 1 is generalized as broadcast phase in which source broadcasts packets, So that it can be heard by relays and destination. Phase 11 relays forward the packet that it receives during phase 1 . Usually phase 1 is similar in all the relaying protocols. Only the processes and procedures in phase 11 distinguish the various relaying protocols. 


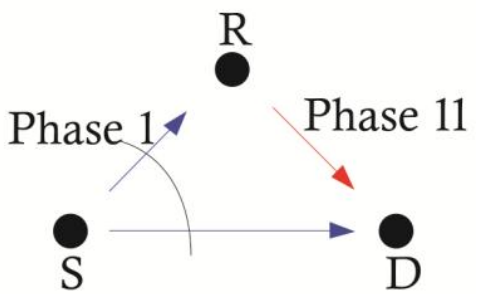

Fig -1: Cooperative diversity scenario for a two user system (S: source, R: relay, D: destination)
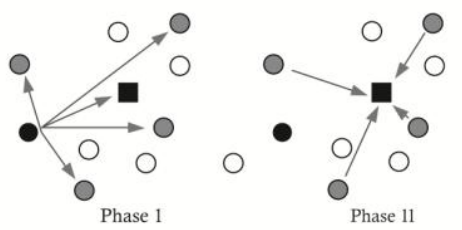

Fig -2: Cooperative diversity scenario for multi user system [5]

This rest of the paper is organized as follows. Section 2 briefly explains the system model. And in section 3 we outline general cooperative diversity protocols. Section 4 presents a literature survey of incremental relaying protocols in detail. Finally in section 5 the paper is summarized.

\section{SYSTEM MODEL}

The system model is considered for single relay cooperative networks. We assume the terminals to operate in time division multiple acces (TDMA) scheme with QPSK signals. The channel is frequency non-selective Rayleigh fading with AWGN (Additive white Gaussian noise) transmission. Let $i$ and $j$ represents two terminal nodes, then $\mathrm{a}_{i j}, \mathrm{n}_{\mathrm{ij}}$ represent the link coefficient and AWGN respectively between the terminals. Each channel coefficient can be modeled as independent complex Gaussian random variable with variance $\sigma_{i j}{ }^{2}$ and mean zero, Given $\gamma_{i j}$ is the instantaneous SNR and $\rho_{i j}$ is the average SNR between terminals $i$ and $j$.Let $\mathrm{R}^{\prime}$ represent the spectral efficiency and $\Delta i j$ is the SNR offsets. The base band equivalent cooperative diversity channel can be modeled as follows:

During phase 1 , the received signals at the destination and relay will be as follows

$$
\begin{aligned}
& Y_{d}=a_{s, d} X_{s}+n_{s d} \\
& Y_{r}=a_{s r} X_{s}+n_{s r}
\end{aligned}
$$

where $\mathrm{X}_{\mathrm{s}}$ represents the source transmitted signal.

The received signal at the destination during phase 11 is

$$
\mathrm{Y}_{\mathrm{d}}=\mathrm{a}_{\mathrm{rd}} \mathrm{X}_{\mathrm{r}}+\mathrm{n}_{\mathrm{rd}}
$$

Where the relay transmitted signal is given by $X_{r}$.

\section{COOPERATIVE DIVERSITY PROTOCOLS}

Cooperative diversity protocols are described in detail by Leneman [1] and Aria Nosratinia and Ahmadreza Hedayat [6]. Cooperative diversity mainly uses fixed, selection and incremental relaying protocols. In this paper, the analysis is performed by considering the transmission of packet data.

In all the cooperative diversity protocols, the transmission of packets is being done in two phases. These operations are illustrated in Fig-1.

- $\quad$ Phase 1: Coordination phase - In this phase, users (source, destination, relay) exchange their own source data and control messages with each other and/or the destination.

- Phase 11: Cooperation phase - In this phase, the users cooperatively retransmit their messages to the destination.

Cooperative diversity transmission is performed by several numbers of protocols such as Fixed relaying, Selection relaying and Incremental relaying. Leneman [1] proposed all these protocols in 2004 and researches in this field are still going on to modify these protocols to obtain a better performance.

- $\quad$ Fixed relaying: The received signals subject to their power constrains will either be amplified, or received signals are re-encoded followed by decoding operation and retransmit the messages.

- Selection relaying: The transmitting terminals are allowed to select a suitable cooperative action based upon the measured SNR between them.

- Incremental relaying: This scheme improves the performance of existing fixed or selection relaying by exploiting limited feedback from the destination and relaying whenever necessary.

\subsection{Fixed relaying}

Fixed relaying is classified into two

- Amplify and forward.

- Decode and forward.

\subsubsection{Amplify and Forward (AF)}

In AF, noisy versions of the transmitted signal from the partner users will be received at every user terminal. The user then amplifies and retransmits this noisy version. The base station proceeds the processing by combining the information sent by the user and partner users, and settles on a final decision about the transmitted bit. Even though the noise is amplified by cooperation, the base station receives two independently faded forms of the signal and can make better decisions on the detection of data.

The expression for probability of outage for amplify and forward scheme is given as

$$
p_{A F}^{\text {out }}=\left(\frac{1}{2 \sigma_{s, d}^{2}} \frac{\sigma_{s, r}^{2}+\sigma_{r d}^{2}}{\sigma_{s, r}^{2} \sigma_{r, d}^{2}}\right)\left(\frac{2^{2 R^{\prime}}-1}{S N R}\right)^{2}
$$




\subsubsection{Decode and Forward (DF)}

Aria Nosratinia and Ahmadreza Hedayat [6] describes decode and forward system as follows. In the decode and forward protocol user tries to decode the partner's data and then proceeds to retransmit the detected data. The base station then combines the data sent by the user and partner, and settles on a final decision about the transmitted bit. The decoding process at the relay terminal removes the noise unlike AF protocol.

The expression for probability of outage for decode and forward scheme is given as

$$
p_{D F}^{\text {out }}=\left(\frac{1}{\sigma_{s, r}^{2}}\right)\left(\frac{2^{2 R^{\prime}}-1}{S N R}\right)
$$

\subsection{Selection Relaying (SR)}

Selection relaying is performed by taking the fading coefficient into account. Optimum selection relaying protocols are discussed by Su and Xin Liu [7]. Fading coefficients [8][9] can be measured accurately in cooperative terminals and they are known to the receivers. So in selection relaying, transmission is adapted in accordance with fading coefficients. Adaptation is done as stated below.

- Measured fading coefficient falls below a certain threshold: The source progresses with its transmission to the destination, in the form of repetition or more powerful codes.

- Measured fading coefficient lies above the threshold: The relay progress with its transmission by either amplify and forward or decode and forward, in order to achieve diversity gain.

The probability of outage for selection relaying scheme is given by

$$
p_{S D F}^{\text {out }}=\left(\frac{1}{2 \sigma_{s, d}^{2}} \frac{\sigma_{s, r}^{2}+\sigma_{r d}^{2}}{\sigma_{s, r}^{2} \sigma_{r, d}^{2}}\right)
$$

\subsection{Incremental Relaying (IR)}

Incremental relaying is developed in order to achieve efficient spectral efficiency and efficient use of degrees of freedom. This particular relaying protocol is discussed in detail in the next section. According to this protocol relay retransmits only if the reception of the packet fails in first phase. IR can be of either IAF (Incremental Amplify and Forward) or IDF (Incremental Decode and Forward) based on the action that is performed by the relay.

The probability of outage for incremental relaying scheme is given by

$$
p_{\text {IAF }}^{\text {out }}=\left(\frac{1}{2 \sigma_{s, d}^{2}} \frac{\sigma_{s, r}^{2}+\sigma_{r d}^{2}}{\sigma_{s, r}^{2} \sigma_{r, d}^{2}}\right)\left(\frac{2^{R^{\prime}}-1}{S N R}\right)^{2}
$$

The performance curve of analytical outage probability for all the diversity protocols discussed so far is given in Fig-3.

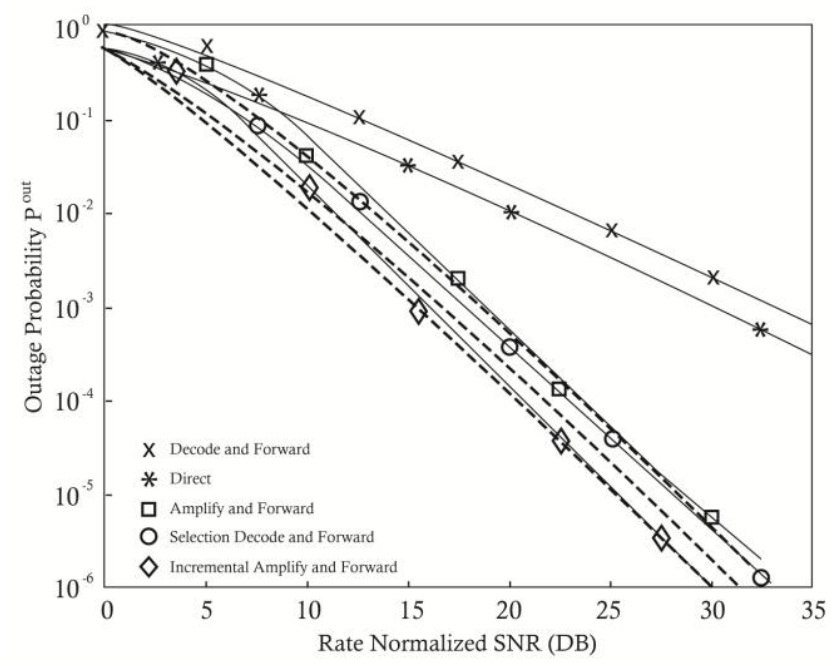

Fig- 3 performance plot of AF,DF, SR, IAF with respect to outage probability and rate normalized SNR[1]

\section{INCREMENTAL RELAYING PROTOCOLS}

Incremental relaying protocol is the key interest of this paper. A number of authors have proposed and analyzed the implementation of this particular protocol. Performance analysis of incremental relaying is still an interesting research field. Leneman [1] Ikki and Ahemed [10] [11], Bastami and Olfat [12], Zhou and Lau [15], Long and Zheng [16], Kuang and Chunjing $\mathrm{Hu}[18], \mathrm{M}$ M Fareed et al.[19] have stated in detail and have evaluated performance of Incremental relaying protocol. like amplify and forward, decode and forward protocol, during phase 1, source mobile node broadcasts the packets to all the other nodes including relays and destinations. But during the second phase the relay retransmits data packets only in the necessary conditions. This means that the relay retransmits only if the reception of the packet fails in the first phase. This is done by providing a limited feedback from the destination, which indicates the success or failure of the packet in the direct transmission phase. Thus the protocol tries to save the spectrum by restricting the relaying process only to the necessary conditions [1].

The feedback information regarding the success or failure in incremental relaying (IF) scheme is determined by the destination is based on either instantaneous SNR (signal to noise ratio) or decoding error. Leneman [1] states that incremental relaying can be viewed as an extension of hybrid ARQ [10]. In ARQ (automatic repeat request) source performs retransmission if destination sends a negative acknowledgement. In incremental relaying the relay performs the retransmission. ARQ protocols for two user cooperative networks have been discussed in detail by Zahng,Wang et al. [13]. They introduced two types of ARQ protocols. Post cooperative and Pre cooperative ARQ protocols. Post cooperative ARQ is a fixed relaying protocol, in which both source and relay take part in every 
ARQ round. Post cooperative ARQ is evaluated by using Alamouti space time coding in [14].

- $\quad$ Step 1: Initially data packet is broadcasted from the source, and then the relay and the destination receives it. Destination then tries to decode the message. If errors are detected while decoding, a negative acknowledgement (NACK) information is sent to source and relay, or else, the acknowledgement (ACK) information is sent.

- Step 2: If the relay receives NACK information in step 1 then it transmits the data packet received during initial broadcasting, or else, the source starts a new transmission.

Zhou and Lau [15] introduced two new protocols for incremental relaying in 2008, and they analysed the protocols by calculating probability of outage and spectral efficiency. Then in the year 2009, H Long, Kan Zheng [16] et al. used fractional incremental relaying protocol as a modification of existing incremental relaying protocols. And Brante, Souza et al. [17] further considered incremental relaying protocol based on fractional incremental relaying and mentioned it as cooperative partial retransmission scheme in their research paper by 2011. Selective fractional incremental relaying (SFIR) is the other protocol which is used in incremental relaying. In SFIR, fractional incremental relaying (FIR) and incremental redundancy (IRD) protocol is the selected scheme as introduced by J. Kuang, C. Hu et al [18] in 2010. And the latest works of incremental relaying is termed as efficient incremental relaying for fading channels as given by M M Fareed, M S Alouini [19] in July 2014.

\subsection{Incremental Selection Amplify and Forward}

\section{(ISAF)}

The ISAF protocol is proposed by Zhou and Lau [15]. According to this protocol there are three feedback messages indicating success, half- success, and failure. So in order to use ISAF the feedback message will be of two bits. In ISAF at the end of phase 1 destination will broad cast one of 3 messages. The 3 feedback messages that are introduced by Zhou and Lau [15] are as following.

I. Success: Success message is generated when the destination decodes the information without any error during phase 1. In this case relay will not perform any retransmission and source proceeds to send the next packet.

II. Half success: If the destination is not able to decode the packet in phase 1 and then if destination determines that doubling the SNR will allow a successful decoding of the packet. In this case a 'half success' message will be broad casted by the destination. So once if the half success message is received then relay will not perform retransmission, instead retransmission will be performed by the source. Combining the signals received in the two phases will double the overall SNR, that is, enhanced by 3 $\mathrm{dB}$, so that the destination will decode the packet without errors.
III. Failure: Suppose if the destination cannot decode the packet successfully in Phase I. Also in addition, it realises that doubling the SNR will not permit a successful decoding of the packet. At that time, it will broadcast a 'failure' message. For this case, during phase 11 the relay amplifies and forwards the information signal that it received in phase 1. The outage probability of ISAF is given below

$$
p_{I S A F}^{\text {out }}=\left(\frac{3}{8 \sigma_{s, d}^{2}} \frac{\sigma_{s, r}^{2}+\sigma_{r d}^{2}}{\sigma_{s, r}^{2} \sigma_{r, d}^{2}}\right)\left(\frac{2^{R^{\prime}}-1}{S N R}\right)^{2}
$$

\subsection{Joint Incremental Selection Relaying (JISR)}

JISR protocol is also proposed by Zhou and Lau [15] with some modifications in the operation when 'failure' message is received as feedback after phase 1. JISR utilizes the channel state information (CSI) between the source and the relay that is known at the relay side in order to take actions during phase 11. JISR protocol operates in a similar manner that of ISAF protocol when 'success' and 'half success' feedbacks are received. In the case of failure feedback relay will perform any of the following actions.

I. Relay encode and forward packet: If relay has successfully decoded the packet that it received during phase 1, will be encoded and forward the packet during retransmission.

II. Relay amplify and forward the packet: If relay has not successfully decoded the packet that it received during phase 1, then it will just amplify and forward the packet during retransmission.

The information regarding whether the relay has been successfully decoded the signal or not during phase 1 , will be determined from the channel condition of the source to the relay.

Outage probability of JISR protocol is given as

$$
p_{J I S R}^{\text {out }}=\left(\frac{3}{8 \sigma_{s, d}^{2}} \frac{\sigma_{s, r}^{2}+\sigma_{r d}^{2}}{\sigma_{s, r}^{2} \sigma_{r, d}^{2}}\right)\left(\frac{2^{R^{\prime}}-1}{S N R}\right)^{2}
$$

Performance analysis plot of ISAF and JISR protocols based on outage probability is given in fig- 4. Also ISAF and JISR is compared with IAF and SDF. 


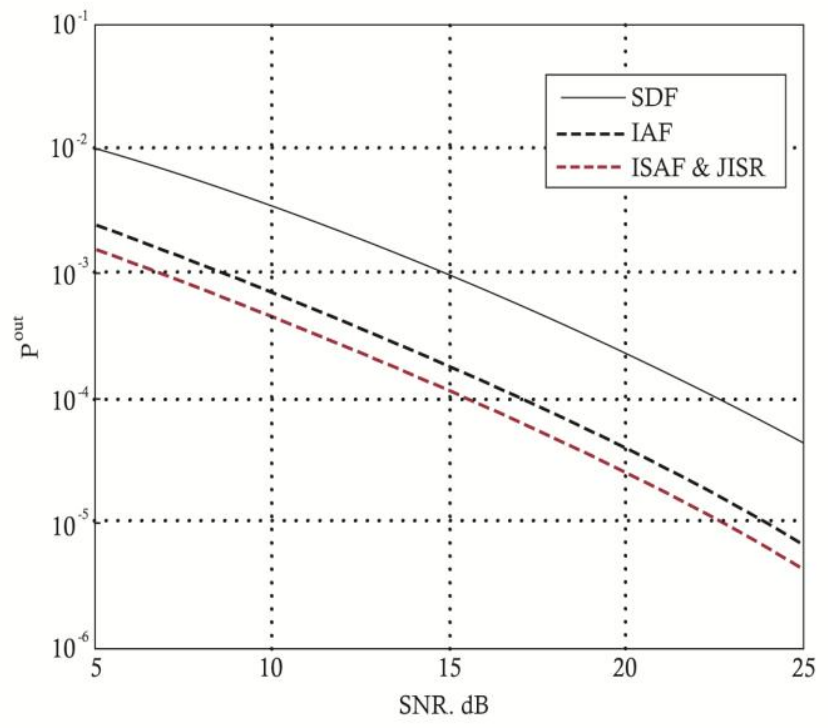

Fig- 4 Performance curve for JISR,ISAF,IAF and SDF cooperative network[15].

\subsection{Fractional Incremental Relaying (FIR)}

In this scheme the idea of partial retransmission HARQ [20], [21] is introduced into incremental relaying protocol in order to achieve spectral efficiency. In the IR protocol so far we have discussed, transmission of the entire packet by relay is a waste of system resource if powerful channel coding is utilized. Fractional incremental relaying (FIR) was proposed by H.Long,K zheng et al. [16] . G G de Oliveira Brante, R.D Souza et al. [17] also studied in detail about FIR in 2011. They termed FIR as cooperative partial retransmission in their work. The idea of FIR is explained as follows.

According to FIR, during phase 11, relay divides the packet into fractions and relay retransmits only a fraction of the original message when the destination requests a retransmission. If the ACK information is received or the maximum number of relaying transmissions is reached, source starts a new transmission. FIR scheme is developed based on the partial retransmission strategy: "When a packet of data needs to be retransmitted, that packet is not repeated entirely. Instead, symbols of that packet are repeated, a few at a time, sequentially, as needed."

The partial retransmission strategy makes more effective use of channel than the full retransmission ARQ strategy [13]. The system operates under the incremental decode and forward (IDF) cooperative protocol using Type-I HARQ with chase combining (CC) [20]. In FIR the code rate changes at each retransmission, and as a consequence, the decoder is more complex in order to support different code rates[8]. Fig-5 illustrates the FIR frame scheme. Detailed protocol description of FIR is given in [16]. H.Long, K zheng et al. [16] done performance analysis and simulation of the protocol and concluded that the FIR protocol makes more efficient use of channel freedom degrees and spends less relay transmission duration.

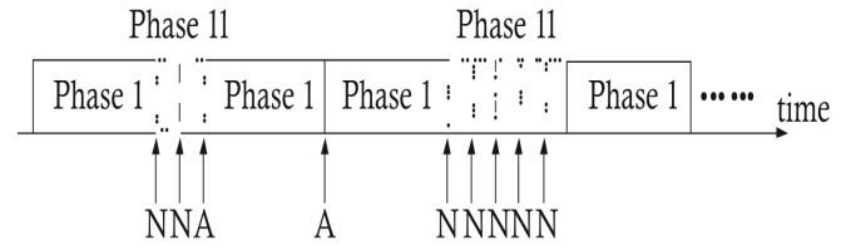

Fig -5: Illustration of FIR scheme [16].

The FIR protocol is described as follows:

Let ' $\mathrm{N}$ ' be the total number of packets. 'L1' denotes the number of coded bits in each packet. And ' $M$ ' denotes the modulation level. ' $\mathrm{N}_{\mathrm{Fir}}$ ' be the number of fractions that a packet is divided into in relay. 'L2' is defined as the number of coded bits in each fraction. ' $i$ ' denotes the number of packets. Where 'i' varies from 1,2..N. 'j' represents fractions into which each packet is divided. ' $\mathrm{j}$ ' varies from $1,2 \ldots ., \mathrm{N}_{\text {Fir }}$.

- $\quad$ Step 1: Initialize $\mathrm{i}=1$. Source transmits a packet with L1/M symbols.

- $\quad$ Step 2: Relay receives the packet and divides it into 'NFir' fractions with $\mathrm{L} 1 /\left(\mathrm{M}^{*} \mathrm{~N}_{\mathrm{Fir}}\right)$ symbols each $\mathrm{L} 2=\mathrm{L} 1 / \mathrm{N}_{\text {Fir }}$.

- $\quad$ Step 3: Destination receives the packet and tries to decode the message.

- 3.1: If errors are detected, the NACK information is sent to Source and Relay.

- 3.2: Otherwise, the ACK information is sent.

- Step4:

- 4.1: If the ACK information is received or the maximum number of relaying transmissions is reached, Source starts a new transmission, go back to Step 1 .

- 4.2: Otherwise, R transmits the $\mathrm{j}$ 'th fraction of the packet just received, $\mathrm{j}=\mathrm{j}+1$, go back to Step 3 .

Let the instantaneous SNR between source and destination be represented by $\gamma_{\mathrm{SD}}$ and equivalent SNR of the received signal in phase 11 be represented by $\gamma_{R}^{e q} \cdot \mathrm{P}_{0}(\gamma)$ and $\mathrm{P}(\gamma)$ represent the approximate $\mathrm{BER}$ (bit error rate) performance under instantaneous SNR $\gamma$ with code word length L1 and the approximate BER performance under AWGN channel respectively. For FIR protocol [16] ,BLER (Block error rate) performance is calculated as

$$
P_{E}^{F I R}=E\left[P_{0}\left(\gamma_{S D}\right) \prod_{i=1}^{N_{F I R}} P_{i}\left(\gamma_{S D}, \gamma_{R}^{e q}\right)\right]
$$

Since average $\operatorname{SNR}\left(\rho_{i j}\right)$ and instantaneous $\operatorname{SNR}(\gamma)$ are related BLER is evaluated against $\rho_{i j}$ in performance analysis (Fig-6). 


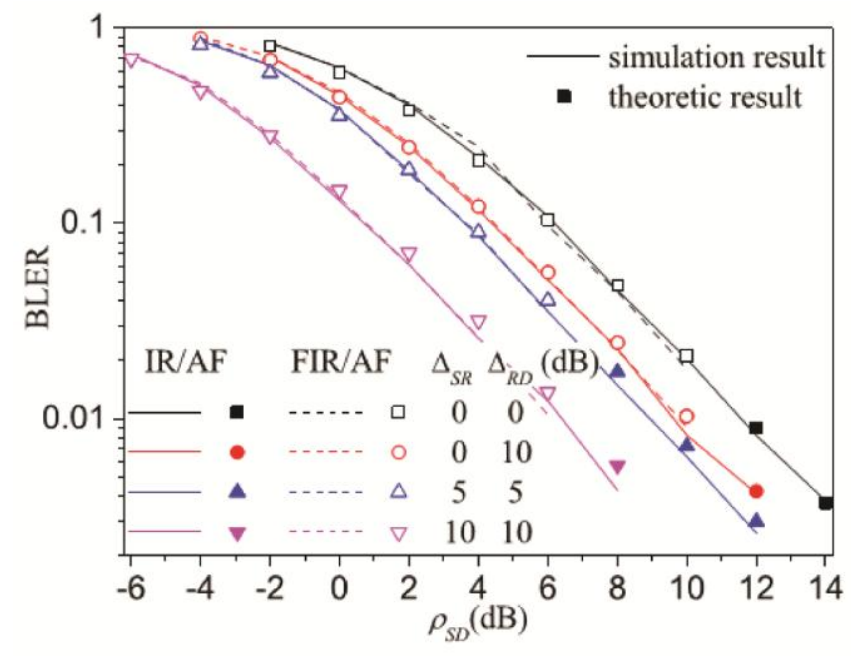

Fig -6: Performance comparison of FIR and IAF for different values of SNR offset [16].

In general if $P_{E}$ is the BLER and $P_{R}$ is the probability for relay transmission then the spectral efficiency is given by

$\eta=\frac{1-P_{E}}{T}$

Where, $\mathrm{T}$ is the transmission duration and is given by

$T=1+P_{R}$

So in FIR Probability of relay transmission $P_{R}$ is smaller compared to all the protocols discussed so far. This is because of the reason that In the FIR protocol, when a packet of data needs to be transmitted by relay node, symbols are transmitted a few at a time as needed instead of the entire packet [15]. Thus the relay transmission duration will be less So according to equation (11) spectral efficiency will be high. Normalized spectral efficiency performance plot is given in fig-4d.

\subsection{Selective Fractional Incremental Relaying} (SFIR)

Selective Fractional Incremental Relaying (SFIR) protocol is the modification of FIR .SFIR is proposed by J. Kuang, C. $\mathrm{Hu}$ et al [18]. SFIR protocol is based on the FIR and the IRD (incremental redundancy) [18] protocols. The FIR and the IRD protocols are similar in the first phase through the source destination link, but different in phase 11. The FIR and the IRD protocols are adaptively selected during phase 11 (retransmission) according to the performance and the overhead .The boundary condition for adaptively selecting FIR and IRD protocols is being calculated on the basis of the instantaneous channel conditions. If the function of instantaneous SNR from source to destination is greater than instantaneous SNR of packet fraction at destination, then phase 11 is proceeded with IRD protocol else FIR is performed. The detailed protocol description of FIR and
IRD is given in [18]. Simulation results in [18] demonstrate that the SFIR protocol makes more efficient use of degrees of freedom compared to the FIR and the IRD protocols.

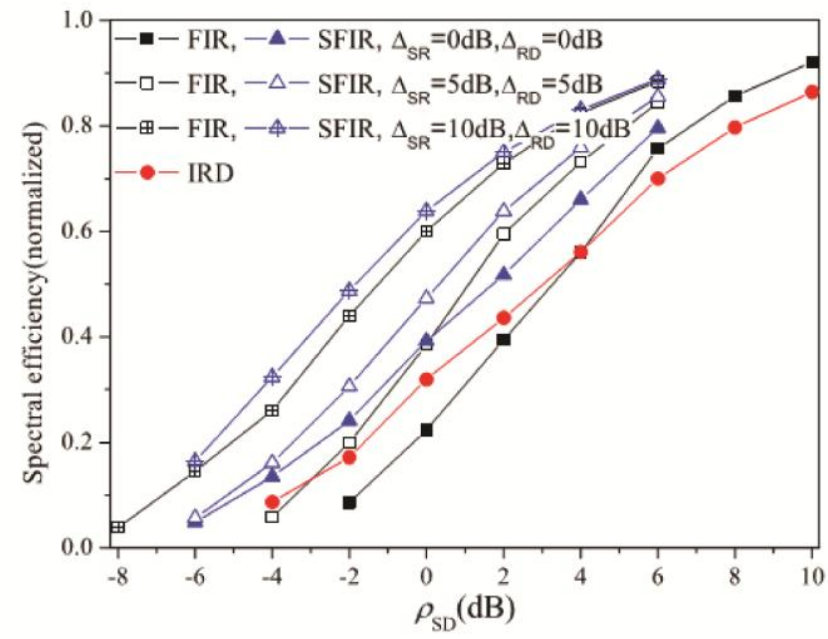

Fig -7: Normalized spectral efficient performance comparison of SFIR with FIR and IRD [18].

Outage probability of SFIR is obtained by modifying the analysis of FIR by taking into account the IRD protocol in required section. The outage probability obtained will be less compared to that of FIR.

Comparison of spectral efficiency performance of IRD,FIR, and SFIR is given in Fig- 7. From the plot it is clear that SFIR out performs IRD and SFIR protocols.

\subsection{Efficient Incremental Relaying (EIR)}

In this scheme a group of $\mathrm{N}$ packets are send from source in phase 1. So unlike all the relaying protocols, according efficient incremental relaying (EIR)[19], a group of packets is sent during phase 1 . At the end of phase 1 the destination sends feedback to the relay in order to indicate that $M$ number of packets out of $\mathrm{N}$ packets is received with low SNR. So in phase 11 the relay forwards only a compartment of weakest packets as instructed and these packets are then combined with the directly received signal using Maximal ratio combining (MRC) and decoded at the destination. Along with this scheme three threshold based schemes are introduced in order to increase the throughput of the scheme. These protocols are introduced by M M Fareed, M S Alouini [19] in 2014 for fading channels.

For the efficient incremental relaying in which the weakest packets are retransmitted, we are considering $\mathrm{N}$ packets out of which M number of packets are weakest ones, the Total packet error rate (PER) is calculated in [19] as

$\operatorname{PER}^{T}=\frac{1}{N} \sum_{i=1}^{M} \operatorname{PER}_{C i}+\frac{1}{N} \sum_{i=M+1}^{N} P E R_{i}$ 
Where $P E R_{c i}$ is the PER of $\mathrm{i}^{\text {th }}$ weakest packet from $\mathrm{i}=1$ to $\mathrm{M}$ that are decoded at destination using MRC and $P E R_{i}$ is the PER of packets from direct link.

Thus the basic scheme under incremental relaying is that the relay forwards the $\mathrm{M}$ weakest packets ranked based on their direct link SNR. The three threshold based schemes other than the basic efficient incremental relaying protocol are as follows.

EIR with threshold at destination (EIR-TD): Packets with the weakest SNR among all the packets are forwarded by the relay based on a threshold SNR at destination. PER of EIR-TD [19] is as follows

$$
P E R^{T D}=\frac{1}{N} \sum_{i=1}^{M} P E R_{R}(i)+\frac{1}{N} \sum_{i=M+1}^{N} P E R_{\text {Dir }} \text { (i) }
$$

Where $P E R_{R}(i)$ is the PER of $\mathrm{i}^{\text {th }}$ weakest packet from $\mathrm{i}=1$ to $\mathrm{M}$ that are decoded at destination using MRC and $P E R_{i}$ is the PER of packets from direct link that are with higher SNR than the threshold SNR $\gamma_{i}$.

EIR with threshold at relay (EIR-TR): Packets at the relay are forwarded only when the received SNR is above a certain threshold. Packet error rate of EIR-TR[19] is

$$
\begin{array}{r}
P E R^{E I R-T R}(i)=F_{S R}\left(\tau_{r}\right) P E R_{N C}(i)+ \\
{\left[1-F_{S R}\left(\tau_{r}\right)\right]\left[P E R_{C O}(i)\right]}
\end{array}
$$

Where $F_{S R}\left(\tau_{r}\right)$ is the probability that the received SNR at the relay is less than the threshold $\tau_{r}, P E R_{N C}(i)$ is the PER with no cooperation and $P E R_{C O}(i)$ is the PER with cooperation.

EIR with threshold at relay and destination EIR-TRD: In this scheme, thresholds are introduced at both the relay and the destination.

The packet error rate and the efficiency of the proposed scheme is calculated to get analytical insight, and provided numerical results to validate the performance of the proposed scheme. The PER [19] at the destination for the $i^{\text {th }}$ packet where $\mathrm{i}=1,2, \ldots, \mathrm{M}$ can be written as

$$
\begin{array}{r}
P E R^{E I R-T R D}(i)=\left[1-P_{r}(c o(i))\right] P E R_{N C}(i)+ \\
P_{r}(c o(i)) P E R_{C O}(i)
\end{array}
$$

Where $P_{r}(\operatorname{co}(i))$ is the probability for cooperation given by

$$
P_{r}(\operatorname{co}(i))=P_{r}\left(\gamma_{S R}>\tau_{S R}\right) P_{r}\left(\gamma_{i} \leq \tau_{D}\right)
$$

$P E R_{N C}(i)$ is the PER with no cooperation, and $P E R_{C O}(i)$ is the PER with cooperation.

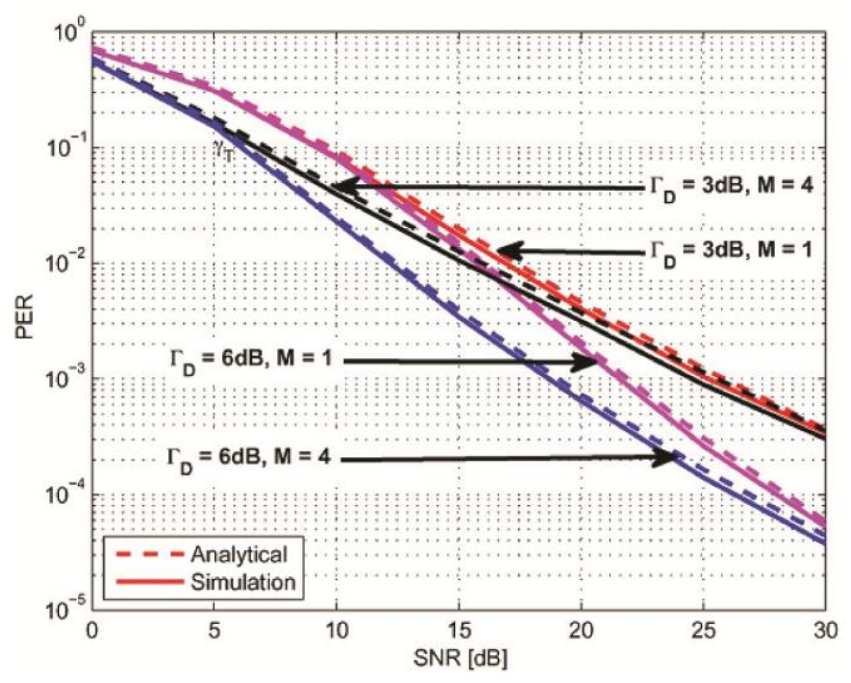

Fig -8: Performance curve of EIR-TD for different values of threshold $\tau_{D}$ for BPSK signalling [19].

Performance analysis curve of EIR-TD protocol with respect to PER and SNR is given in Fig 8 And performance analysis plot of EIR-TR and EIR-TRD can be found in [19].

\section{SUMMARY}

This study contains a brief description of cooperative diversity protocols and a detailed examination of incremental relaying protocols. A detailed discussion of five incremental relaying protocols has been made. The analysis in all the protocols is performed by considering the Rayleigh fading channels and system model of single source relay and destination. ISAF (incremental selection amplify and forward) and JISAF protocols give an improved performance based on outage probability in comparison with traditional incremental relaying protocols. As far as FIR and SFIR are concerned spectrum efficiency is improved. EIR protocol is analyzed by considering three cases EIRTD,EIR-TR and EIR-TRD. So in order to get a better performance in spectral efficiency and outage behavior simultaneously, it has been observed that two or more incremental relaying protocols have to be combined there by completely modifying a new protocol.

\section{REFERENCES}

[1]. J. Nicholas Laneman, D. N. C. Tse, and G. W. Wornell, "Cooperative diversity in wireless networks: Efficient protocols and outage behavior"IEEE Transactions on Information Theory, vol. 50, no. 12, pp. 3062-3080, 2004. [2]. Paul Raj A.J , Gore D A ,Nabar R.U, Bolcskei H “An overview of MIMO communications-a key to Giga-bit wireless " Proceedings of the IEEE(Volume 92,issue 2) [3]. Andrea Goldsmith "Wireless communications" ISBN13 978-0-521-70416-8, Cambridge university press 2005 
[4]. T. M. Cover and A. A. El Gamal, "Capacity theorems for the relay channel,'IEEE Trans. Inform. Theory, vol. IT25, pp. 572-584, Sept. 1979.

[5]. J.Nicholas Laneman, Gregory W. Wornell "Distributed Space-Time-Coded Protocols for Exploiting Cooperative Diversity in Wireless Networks" IEEE Transactions on information theory" Vol. 49, NO. 10, October 2003

[6]. Aria Nosratinia and Ahmadreza Hedayat,Todd E.Hunter "Cooperative communication in wireless networks" IEEE communications magazine, October 2004.

[7]. Weifeng Su, Xin Liu "On Optimum Selection Relaying Protocols in Cooperative Wireless Networks" IEEE Transactions on Communications, VOL. 58, NO. 1, January 2010.

[8]. Theodore S Rappaport "Wireless communications" $2^{\text {nd }}$ ed. Pearson education 2002.

[9]John G.Proakis "Digital communications" $3^{\text {rd }}$ ed.McGraw Hill,Inc 1995

[10]. S.S. Ikki, M H. Ahmed "Performance Analysis of Decode-and-Forward Incremental Relaying CooperativeDiversity Networks over Rayleigh Fading Channels" Vehicular Technology Conference, 2009.

[11]. S.S. Ikki, M.H. Ahmed "Performance analysis of incremental-relaying cooperative-diversity networks over Rayleigh fading channels" Published in IET communications, February 2011 ( Vol .5,Iss 3,pp.337-349).

[12]. A.H bastami, A Olifat "Optimal Incremental relaying in cooperative diversity systems" Published in IET Communications, January 2013 (Vol 7.Iss 2, pp. 152-168)

[13]. C.Zang W.Wang, G . Wei "Design of ARQ protocols for two-user cooperative diversity systems in wireless networks" Elsevier,Science direct Computer communications, pp.1111-1117, 2009

[14]. Branka Vucetic,Jinhong Yuan "Space-Time Coding" John Wiley \& Sons Ltd,2003

[15]. Q. Zhou and F. Lau, "Two incremental relaying protocols for cooperative networks, 'IET Commun., vol. 2, no. 10 , pp. 1272-1278, Nov. 2008

[16]. H. Long, K. Zheng, W. Wang, and F. Wang, "Approximate performance analysis of the incremental relaying protocol and modification," inProc. IEEE 70th VTC-Fall, Anchorage, AK, USA, Sep. 2009, pp. 1-5

[17]. Glauber Gomes de Oliveira Brante, Richard Demo Souza and Marcelo Eduardo Pellenz "Cooperative partial retransmission scheme in incremental decode-and-forward relaying." EURASIP Journal on Wireless Communications and Networking20112011:57.

[18]. Jinghua Kuang, Chunjing Hu, Hang Long, Kan Zheng, Wenbo Wang "Selective Fractional Incremental Relaying Protocol in Cooperative Systems" International Conference on Communications, Circuits and Systems (ICCCAS), 2010 [19]. Muhammad Mehboob Fareed, Mohamed-Slim Alouini, Hong-Chuan Yang "Efficient Incremental Relaying for Packet Transmission Over Fading Channels" IEEE Transactions on Wireless communications, Vol. 13, No. 7, JULY 2014

[20]. Bin Zhao, Matthew C. Valenti "Practical Relay Networks: A Generalization of Hybrid-ARQ" IEEE Journal on selected areas in communications, Vol. 23, No. 1, January 2005
[21]. C Lott, O Milenkovic, E Soljanin, "Hybrid ARQ: theory, state of the art and future directions", in Proc IEEE Information Theory Workshop on Information Theory for Wireless Networks, 1-5 (2007).

\section{BIOGRAPHIES}

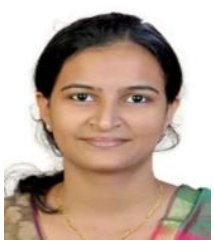

Rinu Titus received the B.Tech degree from Sahrdaya College of Engineering and Technology, University of Calicut in 2012. She is currently pursuing her M.Tech degree in Electronics and Communication Engineering with specialization in Wireless Technology from School of Engineering, CUSAT. Email: rinutitus@gmail.com

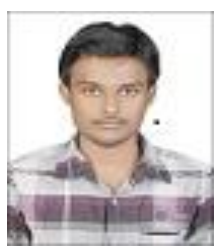

Unnikrishnan $\mathbf{M}$ received the $\mathrm{B}$ Tech Degree in Electronics and Communication Engineering from College of Engineering Trikaripur, Kerala, India, in 2012. He is now pursuing $M$ Tech degree in Electronics and Communication with specialization in Wireless Technology at CUSAT, Kochi, India, Email:unnikrishnanm60@gmail.com

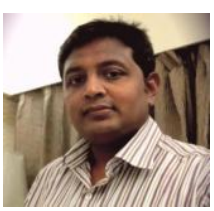

Premkumar C.V received his M.Tech from CUSAT in 2005 June. He is currently serving as a faculty member at CUSAT since 2001. His interested research field is Wireless Communication.

Email: premkumar@cusat.ac.in 